\title{
Matrices de Contabilidad Social y Modelos de Equilibrio General Aplicado elaborados en España a nivel regional
}

\author{
Ángeles CÁMAra SÁnChez ${ }^{a}$, MANUEl Alejandro CARDenete Flores ${ }^{\text {b }}$, \\ José RAMÓn MONROBEL AlCÁNTARA ${ }^{\text {a }}$ \\ a Universidad Rey Juan Carlos, Facultad de Ciencias Jurídicas y Sociales, Paseo de los Artilleros, \\ s/n,28032 Madrid,España.E-mail: angeles.camara@urjc.es, joseramon.monrobel@urjc.es \\ ${ }^{b}$ Universidad Loyola Andalucía, Facultad CC.EE., Campus Palmas Altas, c/ Energía Solar, 1(Edif. \\ G), 41014 Sevilla, España.E-mail: macardenete@uloyola.es
}

\section{RESUMEN}

El objetivo de este artículo es presentar las contribuciones a la modelización del equilibrio general que han sido aplicadas a economías regionales. Se comienza con una descripción de las Matrices de Contabilidad Social construidas para las distintas regiones, para a continuación exponer los distintos Modelos de Equilibrio General Aplicado que se han elaborado utilizando dichas matrices como base de datos. El trabajo pretende ofrecer una visión detallada de cómo ha ido fortaleciéndose este ámbito de investigación a lo largo de los últimos años.

Palabras clave: Matrices de Contabilidad Social, Modelos de Equilibrio General Aplicado, análisis regional.

\section{Social Accounting Matrices and Applied General Equilibrium Models Developed in Spain at Regional Level}

\begin{abstract}
The aim of this paper is to show the contributions to general equilibrium modelling that have been applied to regional economies. We begin with a description of the Social Accounting Matrices constructed for different regions to then expose the different General Equilibrium Models that have been developed using these matrices as database. The work aims to provide a detailed look at how it has strengthened this area of research over the past years.
\end{abstract}

Keywords: Social Accounting Matrices, General Equilibrium Models, Regional Analysis.

Clasificación JEL: C67, C68, D57, D58, R13, R15

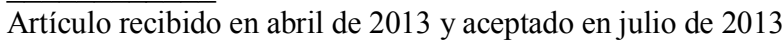

Artículo disponible en versión electrónica en la página www.revista-eea.net, ref. ə-32106 


\section{INTRODUCCIÓN}

El desarrollo de la teoría del equilibrio general que inició Walras en el siglo XIX y el objetivo de muchos investigadores, desde Scarf en la segunda mitad del siglo XX, de llevar dicha teoría a la práctica ha permitido el desarrollo en principio de las Tablas Input-Output, que posteriormente se han completado con las Matrices de Contabilidad Social (MCS). Una vez construida la Matriz de Contabilidad Social se dispone de una base de datos consistente que ha ayudado a la elaboración de Modelos de Equilibrio General.

Este tipo de investigaciones han proliferado tanto a nivel nacional como regional, como posteriormente veremos en el caso de España. Gracias a que la disponibilidad de datos a nivel regional ha aumentado considerablemente en los últimos años, se han podido construir MCS que han permitido realizar una gran variedad de aplicaciones a temas de enorme interés para las economías regionales, como pueden ser los efectos de la aplicación de impuestos y tasas, los efectos de la recepción de Fondos Europeos o los efectos de inversión en energías alternativas.

En los últimos años se han publicado trabajos en los que se ofrecía una revisión de los Modelos de Equilibrio General Aplicado elaborados tanto para la economía española (Cardenete y Llop, 2005) como a nivel internacional (Cardenete, 2009). Este artículo pretende completar la información ofrecida por dichos trabajos, haciendo una revisión de todas las aportaciones que se han realizado a nivel regional dentro de este ámbito de investigación.

El artículo se estructura de la siguiente forma: después de esta introducción, en el epígrafe 2 se describen las Matrices de Contabilidad Social, para seguidamente en el epígrafe 3 detallar las MCS regionales elaboradas en España. Los epígrafes cuarto y quinto están dedicados a los modelos de Equilibrio General. En el cuarto se describen los Modelos Multisectoriales que se elaboran a partir de dichas matrices y en el quinto se detallan los Modelos de Equilibrio General Aplicado construidos para las diferentes regiones españolas. Se concluye con el epígrafe 6 en el que se exponen las principales conclusiones.

\section{MATRICES DE CONTABILIDAD SOCIAL}

Una Matriz de Contabilidad Social (MCS) o Social Accounting Matrix (SAM) es una base de datos que representa de un modo consistente, para un período de referencia, todos los flujos de bienes, servicios y renta entre todos los agentes de una economía; es decir, es una representación matricial a nivel desagregado del flujo circular de la renta. Dichas transacciones reflejan las relaciones existentes entre los agentes económicos, describiendo las operaciones de producción, de distribución, de uso de la renta y de acumulación. Puesto que 
recoge todas las interacciones existentes en una economía, constituye un sistema contable de equilibrio general.

Una MCS proporciona información referida a un año de aspectos tales como la estructura, composición y nivel de la producción, el valor añadido generado por los factores de producción y la distribución de la renta entre los diferentes grupos de economías domésticas. Al incluir tanto las transacciones que se realizan en los Sectores Productivos como las transacciones que se realizan en los Sectores Institucionales así como las transacciones entre Sector Productivo y Sectores Institucionales nos proporciona una visión global de la estructura económica de una nación o región.

Las fuentes de datos principales para construir estas matrices son el Marco Input-Output (MIO) y la Contabilidad Nacional o Regional. Utilizando estas dos fuentes podemos construir una MCS básica que posteriormente desagregaremos en función de las necesidades de modelización. Una de las mayores dificultades a la hora de construir Matrices de Contabilidad Social regionales es la divergencia entre fuentes. Los datos del Instituto Nacional de Estadística (INE) y los datos de los Institutos de Estadística Regionales no siempre son coincidentes, por lo que se hace necesario jerarquizar las fuentes. Las comunidades con Instituto de Estadística propio pueden disponer de mayor cantidad de datos sin necesidad de regionalizar datos nacionales, lo que evita problemas al investigador.

Otro problema con el que nos podemos encontrar es el no disponer de MIO actualizados, en este caso los investigadores han de recurrir a técnicas como el método RAS o el método de Minimización de la Entropía Cruzada ${ }^{1}$ para actualizar al año requerido los datos del último MIO, si bien se deben asumir ciertos errores de ajuste o aproximación propios de estas metodologías.

Las MCS resuelven algunas de las limitaciones informativas de las Tablas Input-Output (TIO). Las TIO centran su información en la cadena de interdependencias productivas de la economía. La ventaja de una MCS es que permite incorporar todas las transacciones económicas que se producen entre todos los agentes en una determinada economía; concretamente, muestra las interrelaciones mutuas entre la estructura de producción, la distribución del ingreso y los patrones de consumo, permitiendo el cierre del flujo circular de la renta.

Las MCS comenzaron a construirse en la década de los sesenta, siendo el pionero Stone (1962), quien desarrolló el concepto de Matriz de Contabilidad Social y quien elaboró el Sistema de Cuentas Nacionales de las Naciones Unidas de 1968 (SCN68), donde se incluyen por primera vez las MCS como un método alternativo de presentación del sistema completo de cuentas.

\footnotetext{
${ }^{1}$ Para una descripción de estas metodologías puede consultarse Bacharach (1970) para el método RAS y Robinson et al. (2001) para el método de Minimización de la Entropía Cruzada.
} 
Dada la importancia que estaban adquiriendo las MCS, en la década de los noventa los sistemas de cuentas nacionales propusieron un método para construirlas. Así lo hace el Sistema Europeo de Cuentas Nacionales y Regionales publicado en 1995 (SEC95) ${ }^{2}$, que es el marco contable para los países de la Unión Europea y es la versión europea del Sistema de Cuentas Nacionales de las Naciones Unidas (SCN93). El SEC95 sustituye al Sistema Europeo de Cuentas Económicas Integradas publicado en 1970 (SEC70) y es de obligado cumplimiento en los países de la Unión Europea para la elaboración de las estadísticas económicas a nivel nacional y regional.

El SEC95 define en su epígrafe (8.134) las matrices de contabilidad social de la siguiente manera: "Una MCS se define como la presentación de las cuentas del SEC en una matriz que explica de forma detallada los vínculos entre una tabla de origen y destino y las cuentas de los sectores. Las MCS se centran, generalmente, en el papel de las personas en la economía, el cual se puede poner de manifiesto mediante desgloses suplementarios del sector hogares y una representación desagregada de los mercados laborales (es decir, distinguiendo varias categorías de personas ocupadas)".

Aunque el SEC95 se decanta por un diseño determinado, con una clasificación bastante amplia para los factores productivos y para el sector de hogares, el formato de las MCS es totalmente flexible y su diseño va a depender del tipo de análisis para el que esté orientado.

En el epígrafe (8.151) del SEC95 se dice: "Dado que una MCS integra tanto los flujos de renta y de gasto como las tablas de origen y destino a nivel macroeconómico, dicha matriz puede utilizarse como modelo para estimar una gran variedad de cuentas. El enfoque MCS es especialmente útil cuando se pretende conciliar información detallada sobre, por ejemplo, la producción y el comercio internacional con datos básicos tomados, por ejemplo, de una encuesta de población activa, una encuesta de presupuestos familiares y una encuesta sobre inversiones en las ramas de actividad. Además, la integración de las cuentas en un marco MCS implica que se puede aplicar el álgebra matricial para equilibrarlas".

E1 SEC95 destaca como características más importantes de la presentación matricial de las cuentas las siguientes:

- La presentación detallada se presta a un tratamiento matemático utilizando el álgebra matricial; esto puede servir de ayuda al equilibrar las cuentas.

- Al presentar un desglose simultáneo de las operaciones interrelacionadas por unidades que realizan los pagos y por unidades que los reciben, resulta

\footnotetext{
${ }^{2}$ Publicado como Reglamente (CE) no 2223/96 del Consejo de 25 de junio de 1996 relativo al sistema europeo de cuentas nacionales y regionales de la Comunidad.
} 
un formato adecuado para desvelar las interrelaciones de los flujos económicos.

- La presentación matricial resulta más concisa que otros métodos de presentación; el pago y el ingreso de las dos unidades que participan en cada operación se reflejan mediante un solo registro contable.

- Puede servir como tabla de referencia para tablas posteriores más detalladas.

- La presentación matricial es una herramienta adecuada para estudiar la flexibilidad del sistema.

En cuanto a su estructura, una MCS es una base de datos, en formato de cuadro de doble entrada, que recoge el flujo de ingresos y gastos de todos los agentes de una economía en un período temporal de referencia. Por convenio, en las filas de la MCS se representan los ingresos monetarios de las cuentas y en las columnas se muestran los respectivos gastos (Tabla 1).

Tabla 1

Identidades macroeconómicas en una Matriz de Contabilidad Social

\begin{tabular}{|l|c|c|c|c|c|c|c|}
\hline & Empresas & Trabajo & Capital & Hogares & Gobierno & Inversión & Sector Exterior \\
\hline Empresas & $T I$ & & & $C$ & $G$ & $I$ & $E$ \\
\hline Trabajo & $W$ & & & & & & \\
\hline Capital & $E B E$ & & & & & & \\
\hline Hogares & & $W$ & $E B E$ & & & & \\
\hline Gobierno & $T i$ & & & $T d$ & & & \\
\hline Ahorro & & & & $A h$ & $D P$ & & $-S C$ \\
\hline Sector Exterior & $M$ & & & & & & \\
\hline
\end{tabular}

Fuente: Elaboración propia a partir de Manresa y Sancho (1997).

A partir de la estructura contable que representa una MCS se pueden observar las diferentes identidades macroeconómicas que verifica. En el nivel más simplificado se pueden representar mediante las siguientes expresiones:

$$
\begin{aligned}
& P I B=C+G+I+(E-M) \quad(\mathrm{PIB}, \text { perspectiva del gasto) } \\
& P I B=W+E B E+T i \quad \text { (PIB, perspectiva de la renta) } \\
& W+E B E=C+A h+T d \quad \text { (Usos de la renta) } \\
& D P=T i+T d-G \quad \text { (Cuentas Públicas) } \\
& S C=E-M \quad \text { (Cuenta Exterior) }
\end{aligned}
$$

donde $P I B$ es el Producto interior bruto, $C$ es el Consumo de los Hogares, $G$ es el Gasto de las Administraciones Públicas, $I$ es la Formación Bruta de Capital, $E$ son las Exportaciones, $M$ son las Importaciones, $W$ son los Sueldos y Salarios, 
$E B E$ es el Excedente Bruto de Explotación, $A h$ es el Ahorro de los Hogares, DP es el Déficit Público, $T i$ son los Impuestos indirectos, $T d$ son los Impuestos directos y $S C$ es el Saldo comercial.

Una MCS puede tomar diferentes formas dependiendo de las cuentas que la integren. En este sentido, es un sistema flexible ya que aunque existe una estructura básica estándar, también permite un alto grado de flexibilidad tanto en el nivel de desagregación como en la elección de la parte del sistema económico en la que se pone mayor énfasis.

Una vez elaborada la MCS básica se puede desagregar de diferentes formas en función de los objetivos de modelización, existiendo MCS con desagregación de Hogares (Moniche, 2003) o MCS con desagregación de impuestos (Cardenete y Sancho, 2003a). Del mismo modo pueden desagregarse las ramas de actividad para estudiar un sector productivo concreto, sectores con una especial importancia a la hora de desarrollar políticas económicas, como puede ser el sector energético (Cámara et al., 2013).

Por otra parte, las posibilidades de ampliación de una MCS facilitan la inclusión de extensiones al sistema de cuentas para incorporar aspectos socioeconómicos tales como información medioambiental $u$ otras magnitudes no monetarias que juegan un papel crucial en la economía. De este modo podemos llevar a cabo análisis que no solo incluyen los aspectos monetarios sino también magnitudes físicas como emisiones de gases contaminantes, cuentas del agua, cuentas de residuos,...

En conclusión, una MCS consiste en una matriz cuadrada, en la que cada una de las filas y columnas, idénticamente determinadas y numeradas (denominadas cuentas de la matriz) representan a cada uno de los agentes, sectores, factores y/o productos que intervienen en la economía que pretende describir. Cada una de las entradas leídas por filas debe interpretarse como ingresos ó outputs para el agente representado en dicha fila, mientras que leídas en columnas se han de entender como gastos o inputs realizados por el agente que se representa en dicha columna. Por tanto, cada elemento de la matriz indica en valores monetarios la transacción por la cual el agente o sector representado en la fila recibe un ingreso procedente del sector o agente representado en la columna.

\section{MATRICES DE CONTABILIDAD SOCIAL REGIONALES}

En España, debido al proceso de descentralización administrativa que se ha vivido en las últimas décadas, han ido apareciendo matrices de contabilidad social de ámbito regional e incluso provincial y local (Cazcarro et al., 2010). La paulatina asunción de competencias por parte de las comunidades autónomas ha generado la necesidad de conocer la estructura de las economías regionales y su evolución en el tiempo, por lo que desde mediados de los años 80 y especialmente durante los años 90 , han comenzado a elaborarse cuentas regionales tanto 
por parte del Instituto Nacional de Estadística (INE) como de las oficinas estadísticas de las diferentes comunidades autónomas.

A partir del año 1986, junto con la Contabilidad Nacional de España (CNE) y vinculada a ésta, el INE elabora la Contabilidad Regional de España (CRE) con el objetivo de ofrecer una descripción cuantificada y lo más completa posible de la actividad económica regional en España (por comunidades autónomas y provincias).

Paralelamente a este desarrollo del sistema de cuentas de España, diferentes instituciones públicas y privadas comenzaron a realizar trabajos en materia contable a niveles regionales. Esta situación ha propiciado el desarrollo de matrices de contabilidad social a nivel regional con el objetivo de realizar análisis de política económica más local.

En España se han elaborado MCS para varias regiones, pero no todas las comunidades autónomas disponen de MCS. La existencia de un Instituto de Estadística propio, como es el caso de Cataluña, Andalucía, País Vasco, Navarra, Castilla-La Mancha o Madrid, es un elemento facilitador de cara a su elaboración. Las distintas comunidades autónomas españolas disponen de oficinas estadísticas, aunque la información que ofrecen varía de unas a otras, habiendo comunidades que aún no disponen de Marco Input-Output propio.

A continuación haremos una breve descripción de todas las MCS regionales que se han elaborado en nuestro país con el fin de tener una visión global de los trabajos que se han realizado en este ámbito de investigación.

La primera MCS regional fue publicada por Curbelo (1986) para Andalucía. Llamada SAMA-80 fue elaborada con los datos de la Matriz Input-Output y la Contabilidad Regional de la Junta de Andalucía y de la Encuesta de Presupuestos Familiares del INE, datos todos del año 1980. La matriz construida por Curbelo contiene 36 cuentas: 23 actividades productivas, 2 factores productivos (capital y trabajo), 8 cuentas de instituciones (5 tipos de hogares, 2 tipos de empresas - con y sin asalariados - y una cuenta combinada de administración central y regional), una cuenta de capital y dos cuentas para los flujos extrarregionales (con Resto de España y con Resto del Mundo).

La segunda MCS para Andalucía fue publicada por Cardenete (1998) referida al año 1990. La SAMAND-90 consta de un total de 31 sectores: 25 sectores productivos, dos factores productivos (trabajo y capital), tres agentes económicos (consumidores, Administración Pública y sector exterior) y una cuenta de ahorro/inversión. Toma como base metodológica la matriz realizada en Cataluña para el año 1987.

Moniche (2003) elabora una MCS para Andalucía referida al año 1995 (MCSAN95). Se trata de una matriz de grandes dimensiones (172*172) que sigue fielmente las directrices del SEC95 quedando una estructura formada por 
10 cuentas, que a su vez se desglosan en varias categorías hasta obtener las 172 cuentas. Aunque esta matriz, a la hora de modelizar, se ha transformado en una matriz de 58 cuentas.

Este proceso de agregación es habitual a la hora de utilizar las MCS construidas como base de datos de diferentes modelos. Dependiendo de qué modelización queramos llevar a cabo tendremos que transformar la MCS agregando o desagregando cuentas. Lo habitual es agregar las ramas de actividad del Marco Input-Output y, en su caso, desagregar alguna de las demás cuentas en función de los objetivos de modelización.

Cardenete y Moniche (2001) elaboraron una MCS de Andalucía referida al año 1995. La SAMAND-95 consta de un total de 35 cuentas: 25 sectores productivos, dos factores productivos (trabajo y capital), un sector representativo de consumidores y empresas, cinco cuentas relacionadas con el sector Administración Pública (cotizaciones sociales de los empleadores, impuestos netos sobre la producción, IRPF, cotizaciones sociales de los empleados y una última cuenta AAPP-ISFLSH ${ }^{3}$, que incluye el resto de los conceptos), una cuenta de ahorro/inversión y el sector exterior.

Una ampliación de la anterior matriz para Andalucía, incluyendo una desagregación mayor de los impuestos, la publican Cardenete y Sancho (2003a). Elaboran una SAM para la economía andaluza con datos del año 1995 a partir de la Tabla Input-Output de Andalucía para el año 1995 (SAMAND-95). Esta SAM tiene 37 cuentas: 25 sectores productivos, dos factores productivos (trabajo y capital), una cuenta de ahorro/inversión, los sectores institucionales (Administración Pública, consumidores, y los diferentes impuestos considerados son las cotizaciones sociales de los empleadores, los impuestos netos sobre la producción, las tarifas, el IVA, el IRPF y las cotizaciones sociales de los empleados) y por último el sector exterior.

Lima y Cardenete (2008) elaboran una MCS para Andalucía referida al año 1999 (SAMAND-99) con un total de 16 cuentas, 10 ramas de actividad, 2 factores productivos y las 4 restantes se corresponden con Consumidores, Ahorro/Inversión, Administración Pública y Sector Exterior. Dicha matriz, junto con las matrices de los años 1990 y 1995, les permite analizar la evolución de la economía andaluza durante una década.

Cardenete et al. (2010) han elaborado una MCS de Andalucía para el año 2000 (SAMAND00) a precios de adquisición, en la que tanto los consumos intermedios como los factores primarios y la demanda final incluyen impuestos indirectos sobre los productos y márgenes comerciales y de trasporte. Para su elaboración se ha utilizado la metodología de minimización de la entropía cru-

\footnotetext{
${ }^{3}$ Instituciones sin fines de lucro al servicio de los hogares
} 
zada $^{4}$. La valoración habitual de las MCS es a precios básicos, dado que habitualmente la información que proporcionan los Institutos de Estadística no contiene tabla de origen a precios de adquisición. La motivación para construir una MCS a precios de adquisición es su utilización como base de datos para modelos de equilibrio genera aplicados (MEGA).

Cardenete y Fuentes (2009) han elaborado una MCS para Andalucía del año 2005 también valorada a precios de adquisición (SAMAND05) y utilizando una metodología distinta a la utilizada en la SAMAND00 basada en tecnologías de transformación Input-Output ${ }^{5}$.

La Comunidad Autónoma de Aragón dispone de una matriz de contabilidad social del año 1999 (SAMA-99) publicada por Flores y Mainar (2009) con el objetivo de hacer un análisis de la economía aragonesa mediante multiplicadores contables. La matriz consta de 36 cuentas: 27 actividades productivas, dos factores productivos (trabajo y capital), una cuenta para Hogares y ISFLSH, una cuenta para Sociedades, una cuenta para el Sector Público, la cuenta ahorroinversión y 3 cuentas para el Sector Exterior (Resto de España, Unión Europea y Resto del Mundo).

Recientemente se han publicado dos MCS para Aragón referidas ambas al año 2005. La SAMA-2005 elaborada por Mainar et al. (2009) con 35 cuentas (26 ramas de actividad, dos factores productivos, tres sectores institucionales, una cuenta de ahorro/inversión y tres cuentas para el resto del mundo) y la MCSA05 elaborada por Pérez y Cámara (2010) con un total de 39 cuentas (26 ramas de actividad, dos factores productivos, tres impuestos, cuatro sectores institucionales, una cuenta de acumulación y tres para el sector exterior).

Ramos et al. (2001) construyeron una MCS referida al año 1995 con las siguientes cuentas: Factor Trabajo, Factor Capital, Sector Privado que engloba a las Empresas, las Familias y las Instituciones de Crédito y Seguro, 16 Ramas de Actividad, Administraciones Públicas, Cuenta de Capital y Sector Exterior; por tanto, con una desagregación en 22 cuentas.

Asturias dispone también de otra MCS para el año 1995 publicada por Argüelles y Benavides (2003) y compuesta de cuatro sectores productivos (agricultura, industria, construcción y servicios), cuatro sectores institucionales (familias, corporaciones, gobierno y sector exterior) y cuatro cuentas más (ahorro/inversión, trabajo, otro valor añadido e impuestos indirectos netos).

Gómez et al. (2004) construyen una MCS para las Islas Canarias del año 1997 con 10 ramas de actividad, 5 factores productivos (tierra, capital, trabajo,

\footnotetext{
${ }^{4}$ Esta metodología consiste en minimizar la distancia entre la matriz original y la matriz buscada, utilizando como restricciones la información estadística disponible para la nueva matriz. Más información sobre este método puede encontrarse en Cardenete y Sancho (2006)

${ }^{5}$ Véase Rueda-Cantuche (2010)
} 
agua y agua de mar) y 4 agentes (Consumidores, Empresas, Gobierno y Resto del Mundo) orientada al análisis del uso del agua.

Baleares también dispone de una MCS publicada por Polo y Valle (2007) y denominada SAM-CAIB97, con un total de 72 cuentas. Las 54 primeras corresponden a las actividades productivas de la Tabla input-output de 1997 y el resto son los factores productivos (trabajo asalariado, cotizaciones sociales y rentas de capital), el sector privado (hogares y sociedades), las Administraciones Públicas (Administración Central y Autonómica) y la cuenta del sector exterior desglosada en hogares no residentes y el resto de operaciones. Presenta un importante desglose de los diferentes tipos de impuestos con un total de 7 impuestos: impuesto sobre la renta de las personas físicas (IRPF) y sociedades (IRS), otros impuestos directos (OID), impuesto sobre el valor añadido (IVA), impuestos ligados a la producción (TP), cotizaciones sociales (CS) y subvenciones de explotación (SB).

Para Canarias, Manrique de Lara (1999) elaboró una MCS del año 1990 actualizando datos del año 1985.

Rubio (1995) elaboró una MCS para Castilla-León referida al año 1985. La SAMCL-85 contiene 10 ramas de actividad, los hogares se han clasificado en 10 grupos en función de su renta, dos factores productivos (trabajo y capital), la cuenta de Administraciones Públicas es única debido a la falta de información para distinguir entre central y regional, el sector exterior sí se desagrega entre resto de España y resto del mundo, las empresas están representadas en una cuenta y la inversión en otra, quedando una matriz con 27 cuentas.

Manresa y Sancho (1997) publicaron una MCS para Cataluña con 34 sectores y elaborada con datos del año 1987. Las 34 cuentas se distribuyen en 23 ramas de actividad, dos factores productivos (trabajo y capital), consumo privado, cuenta de ahorro/inversión, una cuenta para la Administración Pública, cuatro cuentas de recaudación (cotizaciones sociales, impuestos indirectos sobre producción, impuestos indirectos sobre importación e IVA) y dos cuentas para el sector exterior (resto de España y resto del mundo).

La segunda MCS para Cataluña la publicaron Llop y Manresa (1999) referida al año 1994. La SAMC94 se elaboró proyectando a 1994 la Tabla InputOutput de la economía catalana de 1987 utilizando la metodología RAS. Esta matriz consta de un total de 30 sectores: 17 ramas de actividad, dos factores productivos (trabajo y capital), una cuenta del sector privado (hogares y empresas), una cuenta de ahorro/inversión, dos cuentas para la Administración Pública (gobierno central y gobierno regional), tres impuestos indirectos (recaudados por la administración central, recaudados por la administración autonómica e IVA) y cuatro cuentas del sector exterior (resto de España, Unión Europea, resto del mundo e impuestos sobre la importación).

De Miguel et al. (1998) elaboraron la SAMEXT90 para la comunidad extre- 
meña referida al año 1990. Esta matriz tiene 14 cuentas: 8 ramas de actividad, dos factores productivos, una cuenta para el sector privado, una cuenta para las Administraciones Públicas, una cuenta de capital y una cuenta para el sector exterior.

Posteriormente esta matriz fue ampliada y publicada por De Miguel y Manresa (2004) con un total de 35 cuentas. El objetivo para el que se elabora esta matriz es la realización de modelizaciones relacionadas con los diferentes grupos de hogares y la distribución de la renta. Presenta dos factores productivos (trabajo y capital), 11 grupos de hogares desagregados de acuerdo a distintos criterios socioeconómicos, 17 ramas de actividad, una cuenta de ahorro/inversión, una cuenta para el gobierno y tres cuentas para el sector exterior (resto de España, Comunidad Europea y resto del mundo).

Por último, De Miguel et al. (2005) han actualizado al año 2000 la MCS de Extremadura referida al año 1990.

Para Galicia se ha publicado una SAM del año 1999, por Fernández et al. (2006) con un total de 98 cuentas: 33 actividades, 53 productos, dos tipos de hogares (pescadores o no), tres factores productivos (trabajo, rentas mixtas y capital), el gobierno, tres de sus impuestos, la cuenta de ahorro/inversión y por último resto de España y resto del mundo, con el objetivo de analizar la influencia del sector pesquero sobre el conjunto de la economía gallega. En concreto se modeliza un incremento de las exportaciones de dicho sector.

La primera MCS para la Comunidad de Madrid es la SAM-MAD-2000. Ha sido publicada por Cámara (2008) referida al año 2000 y se presenta en dos versiones, la primera sigue la estructura propuesta por el SEC95. Se trata de una matriz agregada con once cuentas que se clasifican en cuatro bloques: cuenta de bienes y servicios, cuentas corrientes (producción, distribución y utilización de la renta), cuentas de acumulación y cuentas del resto del mundo. Esta forma de presentación permite ir obteniendo los sucesivos saldos contables, desde el valor añadido hasta el ahorro y la capacidad o necesidad de financiación de los Sectores Institucionales. Ahora bien, este esquema de presentación no es el más adecuado para la modelización económica, ya que los Sectores Institucionales aparecen agrupados en las diferentes cuentas (asignación, distribución y utilización de la renta). Por ello, la segunda versión con Sectores Institucionales separados en cuentas diferenciadas permite el estudio de impactos en cada uno de los sectores mediante modelos multisectoriales.

Monrobel (2010) ha construido una MCS del año 2002 valorada a precios de adquisición para la Comunidad Madrid, la SAM-MAD02. Para su realización se ha utilizado el método de minimización de la entropía cruzada a partir de la MCS a precios básicos del año 2002 publicada por el Instituto de Estadística de la Comunidad de Madrid. Se estructura en 37 cuentas, con 27 ramas de activi- 
dad, dos factores productivos, tres impuestos, tres sectores institucionales, una cuenta de capital y una cuenta para el resto del mundo.

Por último, Medina (2012) ha elaborado una MCS de la Comunidad de Madrid para el año 2005, la SAM-MAD-2005 con un total de 41 cuentas: 27 ramas de actividad, dos cuentas de ajuste de interior a regional, dos factores productivos, tres impuestos, cinco sectores institucionales, una cuenta de capital y una cuenta para el resto del mundo.

A continuación presentamos en la Tabla 2 las matrices de contabilidad social regionales que hemos descrito en este epígrafe.

Tabla 2

Matrices de Contabilidad Social regionales

\begin{tabular}{|c|c|c|c|}
\hline AUTORES & REGIÓN & AÑO DE PUBLICACION & AÑO DE REFERENCIA \\
\hline Curbelo & Andalucía & 1986 & 1980 \\
\hline Cardenete & Andalucía & 1998 & 1990 \\
\hline Moniche & Andalucía & 2000 & 1995 \\
\hline Cardenete y Moniche & Andalucía & 2001 & 1995 \\
\hline Cardenete y Sancho & Andalucía & 2003 & 1995 \\
\hline Lima y Cardenete & Andalucía & 2008 & 1999 \\
\hline Cardenete y Fuentes & Andalucía & 2009 & 2005 \\
\hline Cardenete, Fuentes y Polo & Andalucía & 2010 & 2000 \\
\hline Mainar y Flores & Aragón & 2009 & 1999 \\
\hline Mainar et al. & Aragón & 2009 & 2005 \\
\hline Pérez y Cámara & Aragón & 2010 & 2005 \\
\hline Ramos, Fernández y Presno & Asturias & 2001 & 1995 \\
\hline Argüelles y Benavides & Asturias & 2003 & 1995 \\
\hline Gómez, Tirado y Rey-Maquieira & Baleares & 2004 & 1997 \\
\hline Polo y Valle & Baleares & 2007 & 1997 \\
\hline Manrique de Lara & Canarias & 2001 & 1990 \\
\hline Rubio & Castilla y León & 1995 & 1985 \\
\hline Manresa y Sancho & Cataluña & 1997 & 1987 \\
\hline Llop y Manresa & Cataluña & 1999 & 1994 \\
\hline De Miguel et al. & Extremadura & 1998 & 1990 \\
\hline De Miguel y Manresa & Extremadura & 2004 & 1990 \\
\hline De Miguel et al., & Extremadura & 2005 & 2000 \\
\hline Fernández-Macho et al. & Galicia & 2006 & 1999 \\
\hline Cámara & Madrid & 2008 & 2000 \\
\hline Monrobel & Madrid & 2010 & 2002 \\
\hline Medina & Madrid & 2012 & 2005 \\
\hline
\end{tabular}

Fuente: Elaboración propia. 
Sin bien, todas las matrices descritas tienen un nexo común en cuanto al número de cuentas, existe una clara diferenciación en la desagregación, por un lado del sector productivo, de acuerdo cada una a su estructura económica regional, y por otro lado, respecto al tipo de análisis que se pretende realizar con la matriz, por ejemplo en la desagregación de los hogares realizada por De Miguel (1998) o en la desagregación de los impuestos, mayor en las matrices con las que se realizan análisis sobre políticas fiscales (Cardenete, 1998, Llop y Manresa, 1999, etc) y menor en los análisis de políticas de inversión (Cámara, 2008, Monrobel, 2010, etc.).

\section{MODELOS MULTISECTORIALES A PARTIR DE MATRICES DE CONTABILIDAD SOCIAL}

La construcción de una MCS tiene dos objetivos fundamentales. En primer lugar, es una base de datos organizada que contiene información desagregada sobre la estructura económica y social de una economía en un año dado. En segundo lugar, constituye una base estadística necesaria para construir modelos que analizan los efectos de determinadas políticas económicas sobre las variables macroeconómicas relevantes.

El tipo de modelizaciones que se han realizado en los últimos años utilizando MCS ha ido variando en función de las diferentes problemáticas a las que se han enfrentado las economías regionales. En la década de los noventa predominaron las modelizaciones de tipo fiscal, debido a las reformas de impuestos como el IRPF o el IVA. En la última década, se han elaborado modelos con el fin de analizar los efectos y la eficiencia de la Política Regional de la Unión Europea, dado que se trata de una de las políticas más importantes a nivel comunitario, tanto por su volumen monetario como por los objetivos perseguidos. Además estos análisis son de gran importancia para las regiones españolas, debido a la posible reducción de estas ayudas en los próximos periodos de programación de los Fondos Estructurales Europeos. Actualmente predominan las modelizaciones de tipo medioambiental, dada la problemática actual relacionada con la contaminación atmosférica y el suministro energético. Prevemos que en los próximos años los investigadores encaminarán sus esfuerzos también a dar luz sobre la actual crisis económica mundial.

Los modelos multisectoriales que se pueden desarrollar utilizando como base de datos una MCS se pueden clasificar en dos tipos: modelos SAM lineales y modelos de equilibrio general aplicado (MEGA).

Los modelos SAM lineales se basan en la obtención de una matriz de multiplicadores que incorpore el conjunto de efectos de interdependencia entre todos los sectores endógenos del modelo. Se trata de una extensión de los modelos input-output incluyendo, además de las relaciones entre los sectores productivos, los flujos que se producen desde los agentes receptores de rentas hacia los 
sectores productivos. Además, permiten endogeneizar los hogares y tratarlos de forma análoga a los sectores productivos. Así, no se analizan únicamente las relaciones intersectoriales sino también los vínculos entre renta de los hogares y gasto. También pueden endogeneizarse el sector público, la inversión o el sector exterior.

El segundo tipo de modelos que se sirven de una MCS como base de datos son los llamados modelos de equilibrio general aplicado. El objetivo principal de los MEGA es conseguir ser una representación empírica que se aproxime lo más posible a las características reales de la estructura económica que es objeto de análisis. Estos modelos se sustentan en una sólida base teórica, la estructura del equilibrio general de Walras (1874).

El impulso definitivo al desarrollo de los MEGA lo dieron los estudios de J. Shoven y J. Whalley (1992) en la década de los 70, que despejaron el camino a los investigadores que han seguido sus pasos. Una de las principales aportaciones de estos autores fue extender el modelo Arrow-Debreu (1954) para incluir aspectos hasta entonces ignorados en los modelos teóricos, como las actividades de las Administraciones Públicas y los intercambios internacionales. Estos primeros modelos se centraban, desde una óptica cuantitativa, en la evaluación de los efectos de la política fiscal en las economías de EEUU y el Reino Unido.

La principal diferencia entre estos modelos y los modelos lineales no es la linealidad de las ecuaciones utilizadas, como habitualmente se considera, ya que los MEGA podrían ser lineales si se consideran las funciones intervinientes lineales. Entendemos que la diferencia radica en que los modelos de equilibrio general aplicado se elaboran a partir del análisis y determinación del comportamiento, que se considera racional, de los diferentes agentes que intervienen en la economía estudiada, la factibilidad tecnológica y las restricciones de los recursos. Sin embargo, los modelos lineales únicamente utilizan las relaciones contables, incluidas o bien en las matrices de contabilidad social o bien en las tablas input-output.

La formulación de un MEGA, de acuerdo con la tradición walrasiana, comienza con la especificación de los bienes y los agentes que integran la economía estudiada. La determinación de estos agentes y bienes tiene como objetivo representar de forma adecuada y simplificada la economía y el problema estudiados. A estos agentes, consumidores y productores, se les supone un comportamiento maximizador de sus respectivas funciones objetivo, que determinan sus planes de actuación, es decir la demanda y oferta de bienes. Junto con estos agentes el modelo se completa con la inclusión de agentes específicos, como son el gobierno y el sector exterior que si bien no tienen porqué jugar un papel esencial en la teoría son imprescindibles en cualquier modelo que pretenda realizar análisis de economías reales. La formulación del modelo teórico finaliza 
con la especificación de las formas funcionales que definen a los agentes de la economía y el comportamiento que tienen dentro del modelo.

Una vez finalizada la determinación del modelo teórico como primer paso de la modelización, para poder realizar los análisis empíricos se debe, a continuación, estimar los valores de los coeficientes de las funciones de oferta y de demanda en todos los mercados y resolver el sistema de ecuaciones resultante para computar el equilibrio de la economía en la situación de referencia inicial (benchmark equilibrium). Este proceso denominado calibración del modelo lo definen Mansur y Whalley (1984) como "el método para las formas funcionales supuestas, que fija el valor de los parámetros desconocidos de forma que el sistema de ecuaciones reproduzca la base de datos como una solución de equilibrio del modelo".

Tal y como acabamos de mencionar, para la determinación de los coeficientes es necesario disponer de una base de datos que refleje de forma consistente y para el periodo de referencia todos los flujos de bienes, servicios y renta entre todos los agentes del modelo. Esta base de datos debemos conseguirla mediante la construcción o la disponibilidad de una matriz de contabilidad social que represente la estructura económica a modelizar. Además de los datos de la matriz también es habitual el uso de otras fuentes que suministren información sobre flujos comerciales, estimaciones de las elasticidades, índices de concentración y otros datos que puedan ser necesarios en el modelo.

Por tanto, tras la calibración, el MEGA replicará la situación de equilibrio de la economía analizada y descrita por la MCS como solución del modelo. Esta réplica nos posibilitará la comparación de este equilibrio inicial con las nuevas situaciones que se le planteen. Estas situaciones surgen mediante la realización de simulaciones en el modelo a través de la introducción de nuevos valores para las variables de política económica o variables exógenas y/o de los diferentes parámetros de las funciones. Se trata, por tanto, de una práctica de estática comparativa, que hace posible la evaluación en el MEGA de nuevas soluciones de equilibrio (counterfactual equilibrium) que pueden derivar de la simulación de diferentes medidas de política económica. La obtención de nuevos equilibrios permitirá visualizar las modificaciones en las principales magnitudes del modelo y así entender o intentar anticipar las consecuencias asociadas a las alteraciones en los escenarios económicos y a las medidas de intervención pública.

En esencia, un modelo de equilibrio general aplicado es un sistema de ecuaciones, generalmente no lineal, que recoge las condiciones de equilibrio general de una economía real, en el que quedan expuestos el funcionamiento de los mercados y las interrelaciones entre los distintos sectores e instituciones económicas y en el que es asumido un comportamiento racional y optimizador de los diferentes agentes, de manera que permiten la inclusión de hipótesis más flexibles que las señaladas en los modelos lineales. 
Actualmente, el desarrollo y aplicación de los MEGA se ha convertido en un método ampliamente utilizado para el análisis de políticas económicas en casi todas las áreas de la economía aplicada, tanto a nivel nacional como internacional. Además en los últimos años se han elaborado una gran cantidad de este tipo de modelos a nivel regional. Nos centraremos en este artículo en hacer un repaso de las principales aportaciones que en este campo se han realizado en nuestro país a nivel regional.

\section{MODELOS DE EQUILIBRIO GENERAL APLICADO REGIONALES}

Como ya hemos señalado en el apartado anterior, la construcción de MCS a nivel regional y la aparición de otras importantes fuentes de información regionales o de Comunidades Autónomas en España posibilitó el inicio en la implementación de modelos de equilibrio general aplicados en el ámbito regional, si bien estos modelos fueron precedidos por algunos modelos lineales. En este sentido, podemos señalar como pionero en España el trabajo de Manresa y Sancho (1997) en el que evalúan la intensidad energética sectorial de la economía catalana mediante el uso de multiplicadores lineales y de un MEGA.

El primer MEGA a nivel regional, elaborado por Cardenete y aplicado a la economía andaluza, no aparece hasta el año 2000. Mediante este modelo se analizan las consecuencias de la reforma del IRPF realizada en el año 1999, presentando dos modalidades, que difieren en la función tecnológica del valor añadido. En una supone una función de coeficientes fijos de tipo Leontief y en la otra permite la sustitución de los factores productivos a partir de una función tipo Cobb-Douglas. El autor utiliza como base de datos la SAM de Andalucía para el año 1995, elaborada en ese mismo trabajo.

Cardenete y Sancho (2003b) presentan dos versiones de un MEGA para Andalucía que se diferencian en la regla de cierre adoptada para describir el comportamiento del sector público. Utilizan este modelo para analizar el impacto que la reforma del impuesto sobre la renta implementada en España podría tener en la región andaluza. Ambas versiones del modelo son calibradas utilizando la SAM de Andalucía del año 1995.

Cardenete (2004) evalúa los posibles efectos que, sobre diferentes agregados macroeconómicos, hogares, actividad productiva y precios, tendría una reducción de las cuotas empresariales a la Seguridad Social para la economía andaluza, adecuándolas a niveles medios europeos. Para ello utiliza un MEGA con base de datos la SAM de Andalucía del año 1995.

André et al. (2005) utilizan un MEGA para simular el efecto de una Reforma Fiscal Ambiental en Andalucía. La reforma supone imponer una tasa a las emisiones de $\mathrm{CO}_{2}$ y $\mathrm{SO}_{2}$ y reducir o el Impuesto sobre la Renta o las cotizaciones de los empleadores a la Seguridad Social, y eventualmente mantener el déficit 
público. Esta aproximación permite evaluar la hipótesis del doble dividendo, la cual establece que este tipo de reformas puede tener beneficios tanto económicos como medioambientales.

Velázquez et al. (2006) analizan los efectos que tendría un incremento en la tarifa del agua del sector agrario sobre la conservación del recurso, la eficiencia en el consumo y la posible relocalización sectorial del mismo. Utilizan un MEGA modificado para introducir las variaciones en la tarifa del agua que analizan mediante la introducción de un impuesto sobre la estructura de producción.

Lima y Cardenete (2007) realizan un análisis de impacto de los Fondos Estructurales Europeos para evaluar sus efectos en producción, precios y renta en Andalucía. Utilizan un MEGA para comparar el escenario de recepción de Fondos regionales con un hipotético escenario donde estos Fondos han sido suprimidos.

Lima y Cardenete (2008) llevan a cabo un análisis de impacto del Fondo Europeo de Desarrollo Regional en Andalucía con el objetivo de evaluar sus efectos en la producción agregada y sectorial, índice de precios y bienestar del consumidor. Los autores realizan simulaciones para tres años representativos: 1990, 1995 y 1999.

Cardenete y Velázquez (2008) introducen la eficiencia en el consumo de agua en el núcleo de un MEGA de la economía andaluza y simulan una mejora de dicha eficiencia, intentando obtener el consumo de agua de los sectores analizados.

Fuentes (2008) ha elaborado un MEGA para Andalucía en el que el principal objetivo es evaluar posibles medidas en política medioambiental, que posibiliten la reducción de emisiones contaminantes y analizar sus efectos sobre la economía regional. Concretamente, la simulación realizada plantea la aplicación de una ecotasa o impuesto medioambiental, con el objetivo de analizar la posibilidad de que exista un doble dividendo, ambiental y no ambiental, derivado de la hipotética reforma fiscal verde simulada.

Cardenete (2009) realiza una aproximación a un escenario de mayor federalismo fiscal a través de un MEGA bi-regional basado en las matrices de contabilidad social de Andalucía y España del año 1995, estudiando los efectos de cambios tanto en los impuestos como en el gasto público, así como sobre ambas economías a nivel de PIB, sectores productivos, precios, recaudaciones impositivas y bienestar social.

Lima et al. (2010) elaboran un MEGA aplicado a la región andaluza en el que plantean un análisis de tipo contrafactual donde simulan cuál hubiera sido el comportamiento de los principales indicadores nominales y reales de dicha economía en el caso de que los Fondos Europeos 2000-2006 no se hubieran reci- 
bido. Tratan así de evaluar tanto la incidencia de las ayudas europeas sobre el PIB, el desempleo y la renta disponible como el grado de efectividad de las intervenciones realizadas.

Cardenete et al. (2010) realizan una estimación del impacto económico regional del desarrollo de las energías renovables basadas en el uso de la biomasa, asumiendo que se alcanza el objetivo fijado en el Plan Andaluz de Sostenibilidad Energética 2007-2013. Para ello utilizan un MEGA construido sobre la SAM de Andalucía actualizada al año 2008.

Cardenete y Hewings (2011) analizan el efecto que un incremento en el precio del agua suministrada al sector agrícola podría tener en la eficiencia de su consumo y la posible reasignación del agua a los sectores productivos restantes. Utilizan un MEGA de la economía andaluza ampliado para incluir emisiones de contaminantes e impuestos ambientales y modificado para introducir las variaciones en el precio del agua que los autores tratan de analizar por medio de un arancel aplicado en la estructura productiva.

Cansino et al. (2011) estiman el impacto en las actividades productivas de aumentar la capacidad de producción de las plantas termosolares instaladas en Andalucía. Se simulan dos escenarios diferentes: primero en base a dos tipos de plantas termoeléctricas que funcionan actualmente y segundo en base a un aumento de los MW instalados para cumplir el Plan Andaluz de Sostenibilidad Energética.

André et al. (2012) ofrecen una nueva lectura de una relación económica clásica: la curva de Phillips a corto plazo. Utilizan un programa multiobjetivo en conexión con un MEGA aplicado a la economía andaluza para determinar la combinación de instrumentos de política económica que proporcionan combinaciones eficientes de inflación y desempleo. Este enfoque da como resultado una versión alternativa de la llamada curva de Phillips eficiente.

Argüelles et al. (2005) elaboran un MEGA de la economía asturiana adaptando un modelo micro-macro para Escocia. La simulación realizada es un estudio del impacto de un aumento de las exportaciones de manufacturas, realizando un análisis de sensibilidad que les ha permitido observar la incidencia que tienen sobre los resultados obtenidos diferentes parametrizaciones del modelo.

Gómez et al. (2004) presentan un MEGA para las Islas Baleares diseñado para analizar las ganancias de bienestar asociadas a una mejora en la asignación de derechos de agua a través de intercambios voluntarios de agua, principalmente entre los sectores agrícola y urbano.

Tirado et al. (2006) utilizan un MEGA de las Islas Baleares para explorar el impacto de aumentar la eficiencia técnica del uso del agua en el sector turístico, demostrando que las medidas de eficiencia en el uso del agua no reducen las presiones económicas sobre los ecosistemas acuáticos. Por consiguiente, el au- 
mento del precio del agua y la reducción de las extracciones de agua son necesarios como medidas adicionales.

El sector turístico balear también ha sido objeto de estudio mediante este tipo de modelos de equilibrio general. Valle (2007) construye un MEGA en el que analiza, por un lado, los efectos que tendría en el conjunto de la economía de Baleares una reducción del turismo de un $10 \%$. Esta reducción es simulada en el modelo mediante la disminución de dicho porcentaje en el consumo de no residentes. En segundo lugar, estudia los impactos económicos de una serie de posibles reformas fiscales (disminución del IRPF, incrementos en las cotizaciones sociales, etc.) en la economía de las Islas Baleares.

Tirado et al. (2010) utilizan un MEGA para Baleares con el objetivo de simular los efectos de un mercado de agua en la agricultura ante escenarios de reducción en la dotación de agua.

El segundo MEGA construido a nivel regional en España analizaba la economía catalana. Con este modelo y sobre la base numérica de una SAM para el año 1990, Llop y Manresa (2004) estudian las consecuencias regionales de una posible reforma en las cotizaciones de empresarios a la Seguridad Social, incorporando distintos supuestos de incidencia de esta figura impositiva entre los empresarios y/o trabajadores.

Llop y Ponce (2012) utilizan un MEGA de la economía catalana para analizar los efectos que los cambios tecnológicos en la agricultura tendrían sobre los indicadores ambientales, sociales y económicos. En concreto, se centran en dos mejoras tecnológicas alternativas: la modernización de los sistemas de transporte de agua en comparación con el aumento de la productividad total de los factores de la agricultura.

Extremadura es la tercera comunidad autónoma cuyo entramado económico ha sido analizado mediante un modelo de equilibrio general aplicado. De Miguel (2003) determina, con este modelo, los efectos que se producirían sobre las principales variables económicas si se suprimieran las subvenciones de explotación dirigidas a la agricultura, subvenciones que en el modelo se consideran inicialmente pagadas por el sector exterior "Comunidad Europea". Este estudio permite aproximarse a un futuro escenario que estaría marcado por la ampliación de la Unión Europea y una posible reducción en los fondos recibidos del sector exterior, que afectarían sin duda a la agricultura extremeña.

De Miguel y Manresa (2008) analizan la importancia de los subsidios agrícolas en la economía extremeña. Presentan un MEGA con el que analizan los efectos económicos de una supuesta eliminación de estos subsidios, planteando diferentes escenarios en los que se consideran rigideces del mercado de trabajo y compensaciones de impuestos.

De Miguel et al. (2009) simulan los efectos sobre la economía extremeña de 
un nuevo impuesto sobre las ventas minoristas de determinados hidrocarburos utilizando un MEGA en el que se contemplan diferentes escenarios en el mercado de trabajo. Incluyen una simulación adicional en la que consideran una hipotética tasa fiscal regional para financiar políticas ambientales, asumiendo constantes los ingresos fiscales.

La última de las comunidades autónomas españolas cuya economía ha sido modelizada a través de un MEGA ha sido la Comunidad de Madrid. Monrobel et al. (2012) presentan un MEGA para la Comunidad de Madrid con el objetivo de estimar el impacto de los Fondos Estructurales 2007-2013. Los efectos derivados de la inyección de los fondos se cuantifican mediante simulaciones realizadas con el modelo desarrollado, centrándose en los objetivos de innovación, investigación, sociedad de la información y desarrollo empresarial.

El primer modelo de equilibrio general dinámico elaborado para el análisis de la economía de una región española ha sido el realizado por Medina (2012), en el que analiza el impacto que supondría para la Comunidad de Madrid implantar un modelo de crecimiento económico basado en la Bioeconomía, entendiendo este como un modelo basado en la inversión en investigación científica, desarrollo tecnológico e innovación. Para ello elabora un MEGA dinámico que permite estimar el impacto sobre la economía regional a lo largo del tiempo mediante la simulación de escenarios modificando al nivel del shock de inversión en cada uno de los sectores implicados.

Por último, Delgado (2012) analiza el impacto sobre la economía andaluza de los Fondos Europeos durante el período 2000-2012, utilizando para cada uno de los períodos analizados diferentes modelos multisectoriales. El período 2000-2006 se analiza a través de un modelo lineal, el 2007-2013 a través de un MEGA y del 2014-2020 a través de un MEGA dinámico.

En la Tabla 3 se presenta un esquema de los modelos regionales descritos anteriormente con sus principales características.

Tabla 3

Modelos de Equilibrio General Aplicado regionales

\begin{tabular}{|l|l|c|l|}
\hline \multicolumn{1}{|c|}{ AUTORES } & REGIÓN & $\begin{array}{c}\text { AÑO DE } \\
\text { PUBLICACION }\end{array}$ & \multicolumn{1}{c|}{ MODELIZACIÓN } \\
\hline Cardenete & Andalucía & 2000 & Reforma del IRPF \\
\hline Cardenete y Sancho & Andalucía & 2003 & Reforma del IRPF \\
\hline Cardenete & Andalucía & 2004 & Efectos de las cotizaciones empresariales \\
\hline André et al. & Andalucía & 2005 & Impuesto ecológico vs reducción IRPF \\
\hline Velázquez et al. & Andalucía & 2006 & Incremento de la tarifa del agua en el sector agrario \\
\hline Lima y Cardenete & Andalucía & 2007 & Fondos Estructurales Europeos \\
\hline Fuentes & Andalucía & 2008 & Introducción de una ecotasa por emisiones de $\mathrm{CO}_{2}$ \\
\hline Lima y Cardenete & Andalucía & 2008 & Fondos Estructurales Europeos \\
\hline
\end{tabular}


Tabla 3 (continuación)

Modelos de Equilibrio General Aplicado regionales

\begin{tabular}{|c|c|c|c|}
\hline AUTORES & REGIÓN & $\begin{array}{c}\text { AÑO DE } \\
\text { PUBLICACION }\end{array}$ & MODELIZACIÓN \\
\hline Cardenete y Velázquez & Andalucía & 2008 & Eficiencia en el consumo de agua \\
\hline Cardenete & Andalucía & 2009 & Federalismo fiscal \\
\hline Lima et al. & Andalucía & 2010 & Fondos Estructurales Europeos \\
\hline Cardenete et al. & Andalucía & 2010 & Energías renovables \\
\hline Cardenete y Hewings & Andalucía & 2011 & Incremento del precio del agua en el sector agrícola \\
\hline Cansino et al. & Andalucía & 2011 & Instalación de una planta termosolar \\
\hline André et al. & Andalucía & 2012 & Desempleo vs Inflación \\
\hline Delgado et al. & Andalucía & 2012 & Fondos Europeos \\
\hline Argüelles et al. & Asturias & 2005 & Exportaciones de manufacturas \\
\hline Gómez et al. & Baleares & 2004 & $\begin{array}{l}\text { Ganancias de bienestar a través del intercambio } \\
\text { voluntario de agua }\end{array}$ \\
\hline Tirado et al. & Baleares & 2006 & Mercado de derechos sobre agua para uso agrícola \\
\hline Valle & Baleares & 2007 & Reformas fiscales. Reducción del consumo turístico \\
\hline Tirado et al. & Baleares & 2010 & Reducción en la dotación de agua \\
\hline Llop y Manresa & Cataluña & 2004 & Reforma en las cotizaciones empresariales \\
\hline Llop y Ponce & Cataluña & 2012 & Políticas de abastecimiento de agua \\
\hline De Miguel & Extremadura & 2003 & Reforma de la Política Agraria Común \\
\hline De Miguel y Manresa & Extremadura & 2008 & Eliminación de subsidios agrícolas \\
\hline De Miguel et al. & Extremadura & 2009 & $\begin{array}{l}\text { Inclusión de un impuesto a determinados } \\
\text { hidrocarburos }\end{array}$ \\
\hline Monrobel & Madrid & 2010 & Fondos Estructurales Europeos \\
\hline Medina & Madrid & 2012 & Investigación y Desarrollo \\
\hline
\end{tabular}

Fuente: Elaboración propia.

Para finalizar, consideramos de interés resaltar cómo han ido evolucionando los problemas que se han analizado con este tipo de modelos, pues nos permite observar cómo han ido variando los focos de interés para los investigadores, lo que está íntimamente vinculado a la problemática económica de cada momento. Si observamos en la tabla anterior la temática de las modelizaciones realizadas podemos constatar la evolución producida en la última década. Durante los primeros años, del 2000 al 2005, predominan los trabajos que analizan políticas fiscales (IRPF, cotizaciones sociales,...). Durante el segundo quinquenio, del 2005 al 2010, aparecen los impuestos medioambientales (ecotasas, impuestos a hidrocarburos,...), trabajos sobre el uso del agua y se analizan los efectos económicos de los Fondos Europeos en varias comunidades autónomas. Por último, en los últimos años, quizá influenciados por la necesidad de un cambio de modelo económico predominan trabajos con una orientación más medioambiental (energías renovables, agua, ...). En la siguiente tabla presentamos los trabajos realizados, organizados según el problema analizado. 


\section{Tabla 4}

Temáticas de los Modelos de Equilibrio General Aplicado regionales

\begin{tabular}{|l|l|}
\hline & Cardenete, 2000 \\
& Cardenete y Sancho, 2003 \\
& Cardenete, 2004 \\
Políticas fiscales: IRPF, ecotasas, cotizaciones,... & André et al., 2005 \\
& Fuentes, 2008 \\
& Cardenete, 2009 \\
& Valle, 2007 \\
& Llop y Manresa, 2004 \\
& De Miguel et al., 2009 \\
\hline \multirow{2}{*}{ Consumo de agua } & Velázquez et al., 2006 \\
& Cardenete y Velázquez, 2008 \\
& Cardenete y Hewings, 2011 \\
& Gómez et al., 2004 \\
& Tirado et al., 2006 \\
& Tirado et al., 2010 \\
& Llop y Ponce, 2012 \\
\hline Fondos Europeos & Lima y Cardenete, 2007 \\
& Lima y Cardenete, 2008 \\
& Lima et al., 2010 \\
& Delgado et al., 2012 \\
& De Miguel, 2003 \\
& De Miguel y Manresa, 2008 \\
& Monrobel, 2010 \\
\hline Energías renovables & Cardenete et al., 2010 \\
& Cansino et al., 2011 \\
\hline Desempleo & André et al., 2012 \\
\hline Exportaciones & Argüelles et al., 2005 \\
\hline & Medina, 2012 \\
\hline
\end{tabular}

Fuente: Elaboración propia.

Consideramos que, dadas las grandes posibilidades de modelización que ofrece esta metodología, en los próximos años se desarrollarán modelos cada vez más sofisticados, que nos permitirán analizar las políticas planteadas tanto a nivel regional como nacional y europeo por parte de los gestores económicos.

\section{CONCLUSIONES}

La construcción de matrices de contabilidad social regionales ha permitido a los investigadores analizar las economías regionales desde un punto de vista global, pues en ellas quedan reflejadas todas las relaciones económicas existentes entre todos los sectores de la economía. Estas bases de datos suponen por sí mismas una fuente de información valiosísima de cara a analizar políticas económicas.

Además de la información macroeconómica que ofrecen, estas matrices son el núcleo de modelizaciones multisectoriales. Basándose en las propias identidades contables de la matriz pueden construirse modelos lineales que, sin nece- 
sidad de más datos externos, nos permiten identificar los sectores más dinámicos de una economía regional, modelizar el impacto de variaciones en la demanda de los sectores productivos, el impacto de cambios en las exportaciones o de medidas fiscales o impositivas.

También, ampliando la matriz con Cuentas Satélite, por ejemplo de emisiones de gases contaminantes, del turismo o del agua, se pueden construir modelos que estudien los efectos de determinadas políticas centradas en estos sectores, como ha sido descrito en el presente trabajo.

Estos análisis podemos extenderlos mediante la elaboración de modelos de equilibrio general aplicado a través de la inclusión de supuestos de conducta de los agentes económicos y sobre el funcionamiento de los mercados de bienes y factores productivos. Estos modelos, además de permitirnos adelantar información sobre los resultados de determinadas intervenciones económicas, mostrando los efectos inducidos sobre los principales indicadores macroeconómicos, también nos permiten analizar los efectos sobre los sectores institucionales, como pueden ser, cambios sobre el bienestar de los hogares, en el mercado laboral, variaciones en el empleo, etc.

En conclusión, se trata de modelos que, dada la gran cantidad de datos que manejan, permiten modelizar todo tipo de políticas económicas como hemos podido ver a lo largo del artículo, modelizando tanto políticas fiscales, comerciales, agrarias, migratorias, etc. como políticas medioambientales o de la Unión Europea.

La recopilación de trabajos que aquí se ha presentado permite tener una visión global de las diferentes temáticas que se han abordado a lo largo del tiempo. Podemos observar que los investigadores no han sido ajenos a los problemas que han preocupado en cada momento a la sociedad, en concreto a las problemáticas particulares de sus regiones, como ha ocurrido en los últimos años en los que los problemas medioambientales han estado cada vez más presentes, por lo que los investigadores han estudiado la manera de introducir datos físicos en sus modelos para reflejar la interacción entre economía y medioambiente. Además debemos señalar que, si bien han predominado los modelos de carácter estático, en el último año con los trabajos de Medina (2012) y Delgado (2012) se ha iniciado un nuevo tipo de modelización para las regiones españolas, los modelos de carácter dinámico, lo que ha permitido presentar los efectos económicos a largo plazo que tiene una determinada política económica.

La diversidad de análisis de impacto que tienen las diferentes modelizaciones que se han descrito en el presente artículo nos hace ser conscientes de la importancia de este tipos de modelos, no solo para los investigadores, sino del interés que tienen o podrían tener para los gobiernos regionales a la hora de tomar decisiones sobre determinadas políticas económicas, al tratarse de un 
instrumento metodológico idóneo para su evaluación ex-ante, on-going y expost.

\section{REFERENCIAS BIBLIOGRÁFICAS}

ANDRÉ, F. J.; CARDENETE, M. A.; VELÁZQUEZ, E. (2005): "Performing an environmental tax reform in a regional economy. A computable general equilibrium approach", The Annals of Regional Science, 39, pp. 375-392.

ANDRÉ, F. J.; CARDENETE, M. A.; LIMA, M. C. (2012): "Using a CGE model to identify the policy trade-off between unemployment and inflation. The efficient Phillips curve", Economic System Research, Vol. 24 (4), pp. 349-369.

ARGÜELLES, M. y BENAVIDES, C. (2003): "Una matriz de contabilidad social para Asturias”, Investigaciones Regionales, 2, pp. 165-171.

ARGÜELLES, M.; BENAVIDES, C.; BILBAO, C. (2005): "Aplicación de un modelo de equilibrio general a la región de Asturias (MEGAAST)", Regional and Sectoral Economic Studies, 5-2, pp. 111-134.

ARROW, K.; DEBREU, G. (1954): Existence of an equilibrium for a competitive economy, Econometrica, 22, 3, p. 265-290.

BACHARACH, M. (1970): Biproportional Matrices and Input-Output Change, Cambridge University Press, London.

CÁMARA, A. (2008): Estimación de la Matriz de Contabilidad Social de la Comunidad de Madrid para el año 2000, Consejería de Economía y Hacienda, Comunidad de Madrid.

CÁMARA, A.; FLORES, M.; FUENTES, P. (2013): "Una Matriz de Contabilidad Social de España para el análisis del sector de las energías renovables", Estadística Española, forthcoming.

CANSINO, J.M.; CARDENETE, M.A.; GONZÁLEZ, J.M.; PABLO-ROMERO, M.P. (2011): "Economic impacts of solar thermal electricity technology deployment on Andalusian productive activities: a CGE approach", The Annals of Regional Science, DOI 10.1007/s00168-011-0471-3.

CARDENETE, M. A. (1998): "Una matriz de contabilidad social para la economía andaluza: 1990", Revista de Estudios Regionales, n 52, pp.137-153.

CARDENETE, M. A. (2000): Modelos de equilibrio general aplicados a la economía andaluza, Tesis doctoral, Universidad de Huelva, editada por Chadwyck-Healey, 2002.

CARDENETE, M. A. y MONICHE, L. (2001): "El nuevo marco input-output y la SAM de Andalucía para 1995", Cuadernos Ciencias Económicas y Empresariales n 41, pp. 13-31.

CARDENETE, M. A. y SANCHO, F. (2003a): "Evaluación de multiplicadores contables en el marco de una matriz de contabilidad social regional", Investigaciones Regionales, $\mathrm{n}^{\circ} 2$, pp. 121-139. 
CARDENETE, M. A. y SANCHO, F. (2003b): "An Applied General Equilibrium Model to Assess the Impact of National Tax Changes on a Regional Economy", Review of Urban \& Regional Development Studies, 15 (1), pp. 55-65.

CARDENETE, M. A. (2004): "Evaluación de una reducción de las cuotas empresariales a la Seguridad Social a nivel regional a través de un Modelo de Equilibrio General Aplicado: el caso de Andalucía”, Estudios de Economía Aplicada, 22 (1), pp. 99-113.

CARDENETE, M. A.; LLOP, M. (2005): Modelos Multisectoriales de Equilibrio General Aplicado en España: una Revisión, Estudios de Economía Aplicada, 23, 2, p. 385404.

CARDENETE, M. A. Y SANCHO, F. (2006): "Elaboración de una matriz de contabilidad social a través del método de entropía cruzada: España 1995", Estadística Española, 48 (161), pp. 67-100.

CARDENETE, M.A.; VELÁZQUEZ, E. (2008): "Ahorro de Agua Mediante la Implantación de Nuevas Tecnologías en el Sector Productivo Andaluz", Revista Innovación, 9, pp. 25-27.

CARDENETE, M. A. (2009): Los modelos de equilibrio general aplicado: una revisión de los principales campos de aplicación a nivel internacional, Revista de Economía Mundial, 23, p. 73-100.

CARDENETE, M. A. y FUENTES, P. (2009): Una estimación de la matriz de contabilidad social de Andalucía de 2005 a precios de adquisición, III Jornadas Españolas de Análisis Input-Output, Albacete.

CARDENETE, M. A.; FUENTES, P.D.; POLO, C. (2010): "Sectores clave de la economía andaluza a partir de la matriz de contabilidad social regional para el año 2000", Revista de Estudios Regionales, $\mathrm{n}^{\circ}$ 88, pp. 15-44.

CARDENETE, M. A.; GONZÁlEZ, J. M.; PABLO-ROMERO, M. P.; ROMÁN, R. (2010): "Impacto de un Incremento de la Capacidad de Generación de Energía a partir Biomasa en Plantas de Cogeneración", Economía Agraria y Recursos Naturales, 10(2), pp. 159-182.

CARDENETE, M. A.; HEWINGS, G.J.D. (2011): "Water Price and Water Sectoral Reallocation in Andalusia. A Computable General Equilibrium Approach", Environmental Economics, 2(1), pp.17-27, 2011.

CAZCARRO, I.; DUARTE, R.; SÁNCHEZ-CHÓLIZ, J. (2010): "Water Consumption Based on a Disaggregated Social Accounting Matrix of Huesca (Spain)", Journal of Industrial Ecology, Vol. 14 (3), pp. 496-511.

CURBELO, J. L. (1986): "MEDEA (Modelo endógeno de desarrollo económico para Andalucía)", Revista de Estudios Andaluces, 7, pp. 13-36.

DELGADO, M.C. (2012): Estimación del Impacto de los Fondos Europeos en Andalucía a través de Modelos de Equilibrio General: 2000-2020, Tesis Doctoral, Universidad Pablo de Olavide, Sevilla.

FERNÁNDEZ, J.; GALLESTEGUI, C.; GONZÁLEZ, P. (2006): "Medición de impactos económicos a partir de una matriz de contabilidad social: el sector pesquero en Galicia”, Revista Española de Estudios Agrosociales y Pesqueros, 22, PP. 41-79.

FLORES, M. Y MAINAR, A. (2009): "Matriz de contabilidad social y multiplicadores contables para la economía aragonesa", Estadística Española, 51 (172), pp. 431-469.

FUENTES, P. (2008): Modelos multisectoriales para la evaluación de políticas medioambientales. Una aplicación a la Economía Andaluza, Tesis doctoral, Universidad de Sevilla. 
GÓMEZ, C.; TIRADO, D.; REY-MAQUIEIRA, J. (2004): "Water exchanges versus water works: Insights from a computable general equilbrium model for the Balearic Island", Water Resources Research, 40, W10502, doi:10.1029/2004WR003235.

LIMA, M.C.; CARDENETE, M.A. (2007): "The effects of European funds on a regional economy: an applied general equilibrium analysis", Applied Economics Letters, 14 (11), pp. 851-855.

LIMA, M.C.; CARDENETE, M.A. (2008): "The Impact of European Structural Funds in the South of Spain", European Planning Studies, 16 (10), pp. 1445-1457.

LIMA, C.; CARDENETE, M.A.; USABIAGA, C. (2010): "Andalucía y el Marco Comunitario de Apoyo 2000-06: Una Evaluación de los Fondos Estructurales Recibidos", Papeles de Economía Española, 123, pp. 102-118.

LLOP, M. y MANRESA, A. (1999): "Análisis de la economía de Cataluña (1994) a través de una matriz de contabilidad social", Estadística Española, vol. 41, n 144, pp. 241 a 268.

LLOP, M. y MANRESA, A. (2004): "The general equilibrium effects of social security contributions under alternative incidence assumptions", Applied Economics Letters, 11 (13), p. 847-850.

LLOP, M. y PONCE, X. (2012): "Agriculture, technological change and environmental sustainability: Looking for a win-win water policy strategy", Document de treball n.17, Universitat Rovira i Virgili.

MAINAR, A., FLORES, M. y DUARTE, R. (2009): Matriz de Contabilidad Social de Aragón 2005. Elaboración y aplicación posterior como base de modelos lineales de multiplicadores, XXXV Reunión de Estudios Regionales, Valencia.

MANRESA, A. y SANCHO, F. (1997): El análisis medio-ambiental y la Tabla input-output: Cálculos energéticos y emisiones de $\mathrm{CO}_{2}$ para la economía de Cataluña, Regidoria de Medi Ambient, Ajuntament de Barcelona.

MANRIQUE DE LARA, C. (1999): Ajuste y actualización de Tablas Input-Output: metodología y aplicación a las Tablas Input-Output de la economía canaria de 1990, Tesis Doctoral, Universidad de Las Palmas de Gran Canaria.

MANSUR, A.; WHALLEY, J. (1984): Numerical specification of applied general equilibrium models: estimations, calibrations, and data. |en| SCARF, H.E; SHOVEN, J. B. Applied General Equilibrium Analysis, Cambridge: Cambridge University Press, p. 69-127.

MEDINA, A. (2012): Análisis del impacto de la inversión en Investigación y Desarrollo mediante modelos multisectoriales como vehículo para implantar la Bioeconomía en la Comunidad de Madrid, Tesis Doctoral, Universidad Rey Juan Carlos, Madrid.

DE MIGUEL, F. J.; MANRESA, A. y RAMAJO, J. (1998): "Matriz de contabilidad social y multiplicadores contables. Una aplicación para Extremadura", Estadística Española, 40 (143), pp. 195-232.

DE MIGUEL, F. J. (2003): Matrices de contabilidad social y modelización de equilibrio general: una aplicación para la economía extremeña, Tesis Doctoral, Universidad de Extremadura.

DE MIGUEL, F. J. y MANRESA, A. (2004): "Modelos SAM lineales y distribución de la renta: una aplicación para la economía extremeña", Estudios de Economía Aplicada, 22-3, pp. 577-603.

DE MIGUEL, F. J.; CARDENETE, M. A.; PÉREZ, J. (2005): "Efectos del Impuesto sobre las ventas minoristas de determinados hidrocarburos en la economía extremeña: un 
análisis mediante modelos de equilibrio general aplicado", Papeles de trabajo del Instituto de Estudios Fiscales. Serie Economía, 12, pp. 1-56.

DE MIGUEL, F. J. y MANRESA, A. (2008): Removal of farm subsidies in a regional economy: a computable general equilibrium analysis, Applied Economics, 40 (16-18), p. 2109-2120.

DE MIGUEL, F.; CARDENETE, M. A. y PÉREZ, J. (2009): "Effects of the tax on retail sales of some fuels on a regional economy: a computable general equilibrium approach", The Annals of Regional Science, Vol. 43 (3), pp. 781-806.

MONICHE, L. (2003): Nuevos desarrollos de las MCS: una aplicación para Andalucía. Instituto de Estadística de Andalucía.

MONROBEL, J. R. (2010): Elaboración de un modelo de equilibrio general aplicado a la Comunidad de Madrid. Estimación del impacto de los Fondos Europeos 2007-3013 en la economía de la región, Tesis Doctoral, Universidad Rey Juan Carlos, Madrid.

MONROBEL, J.R.; CÁMARA, A.; MARCOS, M.A. (2012): "Modelling European Regional Policy 2007-2013: Applied General Equilibrium Analysis of the Economic Impact on the Madrid Region", European Planning Studies, DOI: 10.1080/09654313.2012.722925.

PÉREZ, L y CÁMARA, A. (2010): Estimación de la Matriz de Contabilidad Social de Aragón 2005, Documento de Trabajo $n^{\circ}$ 53, Fundación de Economía Aragonesa (FUNDEAR).

POLO, C. y VALLE, E. (2007): “Un análisis estructural de la economía balear”, Estadística Española, 49 (165), pp. 227-257.

RAMOS, C.; FERNÁNDEZ, E.; PRESNO, M.J. (2001): "Análisis de la economía asturiana a través de la Matriz de Contabilidad Social. Una aplicación a la teoría de los multiplicadores", IV Encuentro de Economía Aplicada, Reus.

ROBINSON, S.; CATTANEO, A.; EL-SAID, M. (2001): "Updating and Estimating a Social Accounting Matrix using Cross Entropy Methods", Economic Systems Research, vol. 13, n० 1, pp. 47-64.

RUBIO, M. T. (1995): Matrices de Contabilidad Social, Junta de Castilla y León, Valladolid.

RUEDA-CANTUCHE, J. M. (2010): Análisis Input-Output estocástico de la economía andaluza. Instituto de Estadística de Andalucía.

SHOVEN, J.; WHALLEY, J. (1992): Applying General Equilibrium. Cambridge: Cambridge University Press.

STONE, R. (1962): “A Social Accounting Matrix for 1960”, A Programme for Growth. Ed. Chapman and Hall Ltd., London.

TIRADO, D.; GÓMEZ, C.; LOZANO, J. (2006): "Efficiency improvements and water policy in the Balearic Islands: a general equilibrium approach", Investigaciones Económicas, Vol. XXX, 3, pp. 441-463.

TIRADO, D.; LOZANO, J.; GÓMEZ, C. (2010): "Economic Regional Impacts of Water Transfers: the Role of Factor Mobility in a Case Study of the Agricultural Sector in the Balearic Islands", Economía Agraria y Recursos Naturales, Vol. 10, 2, pp. 41-59.

VALLE, E. (2007): Modelos Multisectoriales aplicados a la Economía Balear. Palma: Universitat de les Illes Balears.

WALRAS, L. (1874): Elements d'economie politique pure ou Théorie de la richesse sociale, Lausanne: Corbaz. 
VELAZQUEZ, E.; CARDENETE, M.A.; Hewings, G.J.D. (2006): "Precio del agua y relocalización sectorial del recurso en la economía andaluza. Una aproximación desde un modelo de equilibrio general aplicado", Estudios de Economía Aplicada, 24-3, pp. $1-20$. 